\title{
Dislocation-Driven Growth of Two-Dimensional Lateral Quantum Well Superlattices
}

Wu Zhou ${ }^{1,2}$, Yu-Yang Zhang ${ }^{1,3}$, Jianyi Chen ${ }^{4}$, Dongdong Li ${ }^{1}$, Jiadong Zhou ${ }^{5}$, Zheng Liu ${ }^{5}$, Matthew F. Chisholm $^{2}$, Kazu Suenaga ${ }^{6}$, Sokrates T. Pantelides ${ }^{3,1}$, Kian Ping Loh ${ }^{4}$

${ }^{1}$ School of Physical Sciences, University of Chinese Academy of Sciences, Beijing, China.

${ }^{2}$ Materials Science \& Technology Division, Oak Ridge National Laboratory, Oak Ridge, TN, USA.

${ }^{3}$ Department of Physics and Astronomy and Department of Electrical Engineering and Computer Science, Vanderbilt University, Nashville, TN, USA.

${ }^{4}$ Department of Chemistry and Centre for Advanced 2D Materials, National University of Singapore, Singapore.

${ }^{5}$ School of Materials Science and Engineering, Nanyang Technological University, Singapore.

${ }^{6}$ National Institute of Advanced Industrial Science and Technology, Tsukuba, Japan

By stitching different two-dimensional (2D) materials side-by-side or stacking them vertically layer-bylayer, heterostructures can be created with fascinating optical and electronic properties emerging from the hetero-interface due to the coupling between the different $2 \mathrm{D}$ components. Creating multiple heterointerfaces by constructing 2D multi-heterojunctions or superlattices provides a promising way to tailor the physical properties and inducing new functionalities in 2D materials. This has recently been an intensely pursued topic in 2D materials research. However, fabricating 2D lateral multi-heterostructures or superlattices with width smaller than $5 \mathrm{~nm}$, a regime where quantum size effect would come into play, remains a major challenge.

Using low-voltage aberration corrected scanning transmission electron microscopy (STEM) imaging, we presented a systematic study of lateral 2D semiconductor heterostructures with and without lattice mismatch, where strain distribution and atom-by-atom chemical mapping at the lateral interfaces can be obtained by quantitative analysis of the STEM images. We show that for 2D lateral heterostructures where the two monolayer components have similar crystal structure and negligible lattice mismatch, such as $\mathrm{WS}_{2} / \mathrm{MoS}_{2}$ or $\mathrm{WSe}_{2} / \mathrm{MoSe}_{2}$, lateral epitaxial growth can lead to fully coherent and atomically abrupt interface [1].

In contrast, superlattice of sub-2-nm-wide quantum wells can be created when lateral epitaxy growth is applied to 2D components with considerable lattice mismatch, e.g. between a selenide monolayer (WSe $\mathrm{W}_{2}$ or $\left.\mathrm{MoSe}_{2}\right)$ and a sulfide monolayer $\left(\mathrm{WS}_{2}\right.$ or $\left.\mathrm{MoS}_{2}\right)$ (Figure 1). We show that strain relaxation at such lateral mismatched interfaces often leads to misfit dislocation arrays, which are highly active during the high temperature chemical vapor deposition (CVD) growth for the heterostructures. Insertion of metal and $\mathrm{S}$ atoms into the dislocation cores induces dislocation climb while concomitant selective substitution of Se atoms around the dislocation core by $\mathrm{S}$ atoms, driven by the local strain field, lead to growth of $\mathrm{WS}_{2}$ and $\mathrm{MoS}_{2}$ quantum-well arrays embedded in the $\mathrm{WSe}_{2}$ and $\mathrm{MoSe}_{2}$ monolayers (Figure 2). The quantum wells obtained using this mechanism form fully coherent lateral interfaces with the selenide monolayer matrix, and have a uniform width controlled by the strain field around the misfit dislocation cores and their separation controlled by the lattice parameters of the two 2D components [2]. Preliminary monochromatic electron energy-loss spectroscopy (EELS) analysis shows that the optical properties of such quantum well superlattices are considerably different from their parent 2D components. Such misfitdislocation-driven growth should be applicable to different combinations of $2 \mathrm{D}$ monolayers with lattice 
mismatch, paving the way to a wide range of 2D quantum-well superlattices with controllable band alignment and nanoscale width [3].

References:

[1] Y. Gong et al., Nature Materials 13 (2014), p. 1135-1142

[2] W. Zhou et al., Science Advances (2018) in press.

[2] W.Z. acknowledges support from the Natural Science Foundation of China (51622211) and the CAS Key Research Program of Frontier Sciences. This research was also sponsored by the U.S. Department of Energy, by the McMinn Endowment, and by the Singapore National Research Foundation under NRF RF Award No. NRF-RF2013-08, Tier 2 MOE2016-T2-2-153 and MOE2015-T2-2-007.
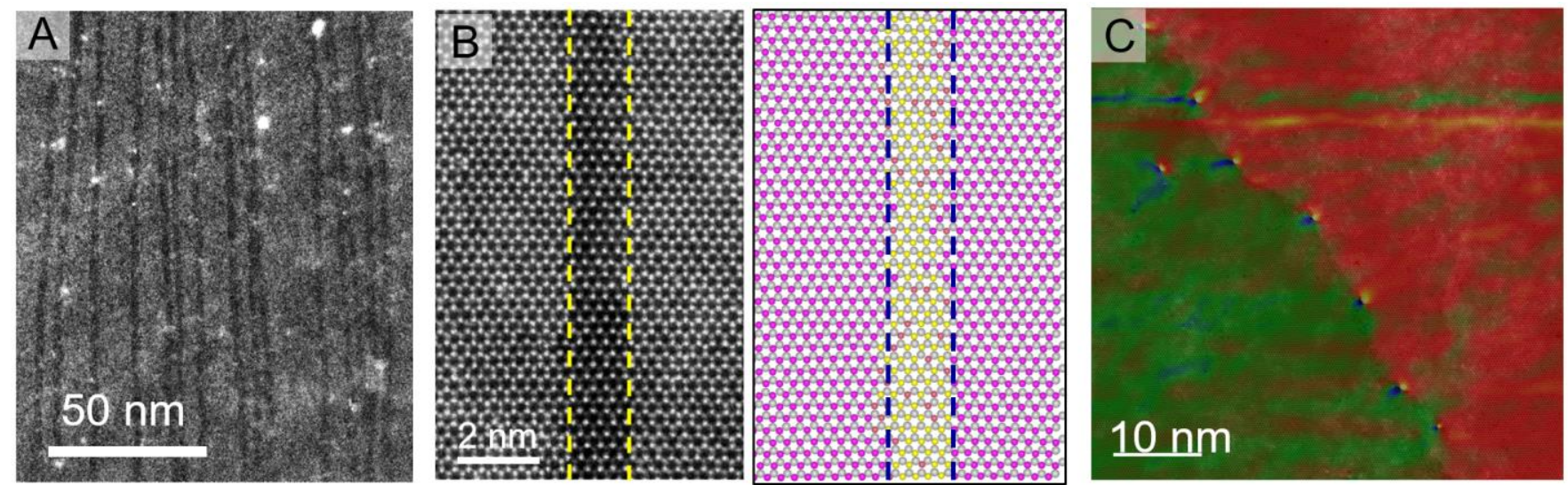

Figure 1. (A) Low magnification STEM-ADF image showing the formation of $\mathrm{MoS}_{2}$ quantum well superlattice in monolayer $\mathrm{MoSe}_{2}$. (B) High resolution STEM-ADF image of a $\mathrm{WS}_{2}$ quantum well and the corresponding atomic structural model. The dashed lines highlight the coherent lateral interface along the armchair direction. (C) Strain distribution of a $\mathrm{WS}_{2} / \mathrm{WSe}_{2}$ lateral interface, obtained from GPA analysis of STEM-ADF image, showing the formation of periodic dislocation array before the formation of $\mathrm{WS}_{2}$ quantum wells.
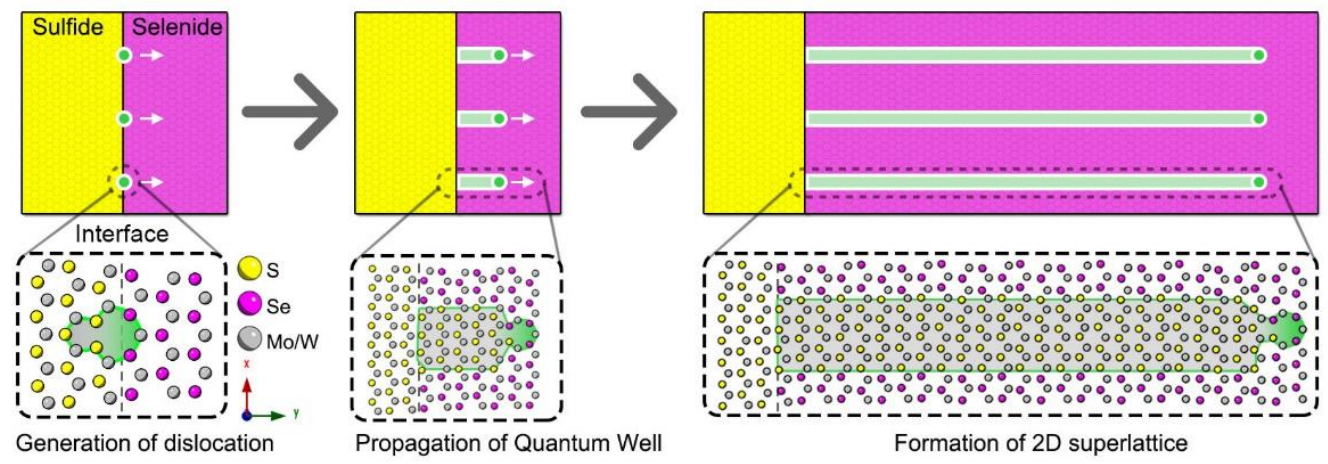

Figure 2. Schematic showing the formation of periodic dislocation array, dislocation-driven growth of $\mathrm{WS}_{2}$ quantum wells, and formation of $2 \mathrm{D}$ quantum well superlattice in the $\mathrm{WS}_{2} / \mathrm{WSe}_{2}$ lateral heterostructure. 